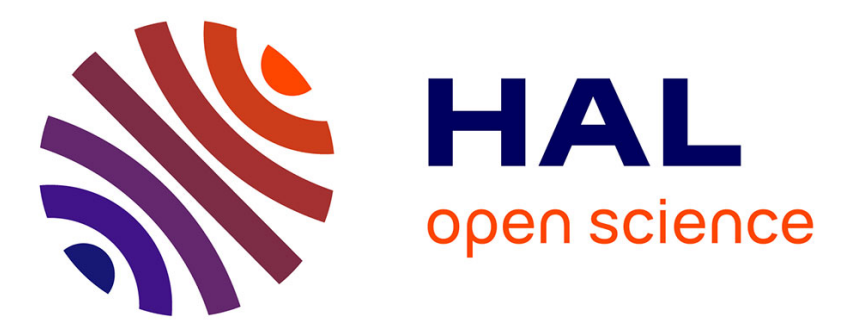

\title{
Heurs et malheurs de la raison dans Les secrets de Monsieur Synthèse (1888), roman d'anticipation scientifique par Louis Boussenard \\ Stéphanie Dord-Crouslé
}

\section{- To cite this version:}

Stéphanie Dord-Crouslé. Heurs et malheurs de la raison dans Les secrets de Monsieur Synthèse (1888), roman d'anticipation scientifique par Louis Boussenard. L'esprit créateur, 2017, L'esprit (dé)réglé: Literature, Science, and the Life of the Mind in France, 1700-1900, Volume 56, Number 4, Winter 2016, pp.104-117. 10.1353/esp.2016.0045 . halshs-01148812

\section{HAL Id: halshs-01148812 \\ https://shs.hal.science/halshs-01148812}

Submitted on 1 Feb 2017

HAL is a multi-disciplinary open access archive for the deposit and dissemination of scientific research documents, whether they are published or not. The documents may come from teaching and research institutions in France or abroad, or from public or private research centers.
L'archive ouverte pluridisciplinaire HAL, est destinée au dépôt et à la diffusion de documents scientifiques de niveau recherche, publiés ou non, émanant des établissements d'enseignement et de recherche français ou étrangers, des laboratoires publics ou privés. 
«Heurs et malheurs de la raison dans Les secrets de Monsieur Synthèse (1888), roman d'anticipation scientifique par Louis Boussenard »

Stéphanie Dord-Crouslé

L'Esprit Créateur, Volume 56, Number 4, Winter 2016, pp. 104-117 (Article)

Published by Johns Hopkins University Press

DOI: $\underline{\text { https://doi.org/10.1353/esp.2016.0045 }}$

Version « auteur » de l'article

[p. 104]

\section{Heurs et malheurs de la raison dans Les secrets de Monsieur Synthèse (1888), roman d'anticipation scientifique par Louis Boussenard}

Stéphanie Dord-Crouslé

On se propose ici d'interroger la vie de l'esprit au XIX ${ }^{\text {e }}$ siècle en produisant une étude de cas, au sens clinique (un peu) mais surtout littéraire du terme. Il s'agira de procéder à l'autopsie spirituelle d'Élias-Alexander Synthèse, mieux (ou un peu moins mal) connu sous le nom de «Monsieur Synthèse », personnage quasi éponyme du roman de Louis Boussenard intitulé Les secrets de Monsieur Synthèse ${ }^{1}$.

Cet individu présente la particularité de posséder un esprit clivé, bifrons, au sein duquel s'affrontent une lumineuse rigueur scientifique et d'inquiétantes dimensions occultes. La construction littéraire qu'opère Boussenard de ces données conflictuelles illustre la confrontation de deux univers que la confiance contemporaine en la science aspire alors à voir se rejoindre, mais dont l'appariement ne va pas sans résistances. Les secrets de Monsieur Synthèse est donc un roman représentatif d'une tendance de fond qui s'affirme à la fin du XIX siècle et qui pousse à la «scientification du merveilleux ${ }^{2}$ ». Mais le déroulement de l'intrigue ainsi que sa conclusion semblent se dérober à cette logique agrégative : le merveilleux fait de la résistance et les péripéties de cette lutte peuvent être appréciées au prisme des mutations de l'esprit de Monsieur Synthèse.

Pour les suivre, on s'intéressera d'abord au tableau que Boussenard dresse d'un esprit avide de repousser toujours plus loin les limites de la science et à qui rien ne semble pouvoir résister. Cet esprit se révèle pourtant parcouru de failles : il entretient un rapport pathologique et potentiellement pathogène à la science. 
Enfin, on s'interrogera sur le rôle que l'auteur fait jouer à un personnage mystérieux nommé Krishna. Ce pundit ou brahmane (qui pourrait n'être qu'un pur esprit) semble cependant suggérer qu'il y a une autre manière de penser l'association de la science et du merveilleux.

Le personnage de Monsieur Synthèse est d'abord caractérisé par le rapport particulier et quasi charnel qu'il entretient avec la science. Son patronyme est la première manifestation de cette union intime puisqu'il le tient d'un ancêtre qui, « au lieu de perdre son temps aux billevesées enfantées par le cerveau de ses contemporains, conçut l'idée géniale de tenter la reproduction

[p. 105]

des corps composés » (35). À l'origine du système qui permit l'essor de la chimie moderne, c'est-à-dire la synthèse chimique, il « conserva ce nom qui en valait bien un autre et le transmit à ses descendants » (36). C'est donc dans cette branche disciplinaire que le personnage, "synthèse vivante, [...] résultante de tous ces efforts séculaires, [...] incarnation de dix générations d’obscurs et acharnés travailleurs » (36), a particulièrement œuvré, lui qui a " hérité non seulement du nom et des travaux de [ses] ancêtres, mais encore d'une propension irrésistible qui [le] pousse vers l'étude des sciences, et notamment de la synthèse chimique... » (36).

Cette hérédité et ce bagage familial initial ont ensuite été complétés au fil des années par des études continues et approfondies, menées en particulier du côté des sciences naturelles et de la théorie de l'évolution :

Depuis longtemps la physique et la chimie biologique, encore en enfance aujourd'hui, bien que professées par des maîtres éminents, n'avaient plus de secrets pour lui. La physiologie transcendante qui étudie la production de la pensée, le mécanisme des sensations, les rapports intimes du moral avec la matière, ou du cerveau avec ses fonctions, etc., tout cela lui était connu depuis longtemps, ainsi que les théories de la descendance, à peine formulées par les chefs d'école, et dans tous les cas empyriquement [sic] déduites. (442)

Aussi n'y a-t-il rien d'étonnant à ce que, aux yeux du préfet de police qui vient l'interroger sur ses inquiétants projets au tout début du roman, Synthèse apparaisse sous « le masque imposant de Darwin, popularisé depuis longtemps par les publications illustrées » :

C'est bien là le front immense de l'illustre physiologiste anglais. Un front légèrement fuyant comme celui d'un rêveur, qui brusquement s'élargit en deux énormes protubérances latérales, et semble se prolonger jusqu'à l'occiput, en une dernière et plus énorme voussure, doublant pour ainsi dire la capacité de la boîte crânienne. (21) 
La nature a donc doté le savant de dispositions physiologiques appropriées et hors-normes qui doivent lui permettre de mener à bien son ambitieux projet : installer, au beau milieu de la mer de Corail, un « laboratoire gigantesque » dont le sol, «improvisé de toutes pièces », " émergera du sein des eaux » et permettra «d'opérer l'évolution de toute la série animale depuis la monère jusqu'à l'homme» :

Je prétends prendre une simple cellule organique, la mettre dans un milieu de développement essentiel à son évolution, favoriser par des agents énergiques et spécialement appropriés, cette évolution, de façon à reproduire, en moins d'une année, tous les phénomènes de transformation qui se sont opérés depuis le moment où la vie organique, représentée par cette cellule, s'est manifestée sur la terre, jusqu'à l'apparition de l'homme. (56)

[p. 106]

Chimiste et expérimentateur de génie, "plus savant à lui seul que la Bibliothèque nationale » (14), Monsieur Synthèse a une confiance aveugle dans le progrès ${ }^{3}$. Pour lui, rien n'est impossible à la science et il accueille avec une grande satisfaction la confession qu'il finit par arracher à un préfet de police d'abord fort circonspect: "Ma foi, Monsieur, nos modernes chercheurs nous saturent de tant de merveilles, qu'en notre siècle de vapeur, d'électricité, de téléphones, de phonographes, d'aérostats dirigeables, je commence à croire que la science peut tout réaliser » (26).

L'esprit de Monsieur Synthèse est donc naturellement tout entier tourné vers ce qui peut favoriser le progrès et particulièrement soucieux des conditions dans lesquelles celui-ci peut se développer : on ne peut pousser plus loin que lui «le scrupule de la précision en matière d'expérience» et il fait preuve d'une «susceptibilité excessive à l'endroit de tout ce qui n'est pas rigoureusement scientifique » (392).

Aussi son ultime projet paraît-il se mettre en place peu à peu, exactement selon les plans qu'il avait préalablement arrêtés :

Pendant cette période déjà longue de trente jours, pas la moindre avarie aux appareils qui fonctionnent avec une régularité, une précision merveilleuses, pas la moindre interruption dans l'enchaînement mystérieux des êtres qui apparaissent successivement sous la coupole de verre; pas le moindre accroc à l'évolution artificielle si audacieusement tentée par le vieux savant !

Aussi, le bonheur goûté par Monsieur Synthèse, tout en étant d'essence uniquement scientifique, n'en est-il pas moins absolu.

Qui concevra, du reste, ces joies discrètes, austères aussi, mais singulièrement passionnantes, de chercheur obstiné, assistant, minute par minute, à l'éclosion de choses prévues? (284) 
Nulle surprise, aucun événement inattendu ne vient perturber le déroulement de l'expérience. Cette expérimentation contrôlée de bout en bout ne fait que répéter, quant au succès dont elle semble devoir être couronnée, les réussites que Synthèse a déjà connues dans d'autres domaines :

Déjà imprégné en naissant, par atavisme, de l'esprit scientifique de ces ancêtres qui, indépendamment de cellules cérébrales héréditairement accommodées à l'étude, lui avaient légué d'inestimables trésors, Monsieur Synthèse avait pu, grâce à ce prodigieux apport, résoudre des problèmes dont le seul énoncé ferait la stupeur de nos maîtres les plus illustres. (442)

Synthèse est donc aussi un visionnaire de génie qui a fait progresser la science et a imaginé des développements qu'il n'est pas forcément lui-même en mesure d'assurer, mais dont on peut être certain qu'ils seront réalisés dans le futur. En particulier, d'ici quelques milliers d'années, un «projet de correspondance entre la Terre et Mars » (27) verra, selon lui, le jour grâce à la modification de l'orbite de notre planète ${ }^{4}$.

[p. 107]

Ce scientifique dont l'extraordinaire productivité n'a d'égale que la prodigieuse inventivité est à lui-même son premier terrain d'expérimentation. En effet, son esprit a acquis la complète maîtrise de son corps et de ses principaux mécanismes physiologiques. Comme il l'explique à un préfet de police quelque peu abasourdi, il a résolu les deux problèmes essentiels de l'existence matérielle : la nourriture et le sommeil.

Depuis plus de trente ans, Monsieur Synthèse ne mange plus à la manière du commun des mortels mais ingère quotidiennement, à heure fixe, dix pilules qui sont composées de « matières alimentaires chimiquement pures » (34). C'est grâce à ses connaissances personnelles en chimie, et en particulier à sa maîtrise des règles de la composition des corps simples, que le scientifique a pu parvenir à cet intéressant résultat. En ce qui concerne son repos, il a recours à des savoirs très anciens (puisque la pratique en est « connue en Orient, depuis des époques immémoriales » [38]), mais qui ont été popularisés par les «très remarquables expériences [...] faites récemment à la Salpêtrière par le professeur Charcot, et à Nancy par le professeur Bernheim » (37). Ainsi, depuis 45 ans, grâce au procédé de l'hypnose et de son corollaire la suggestion, Monsieur Synthèse a remplacé le sommeil naturel par un sommeil de nature hypnotique. Comme «les fakirs hindous qui s'hypnotisent à volonté » (38), il provoque son propre sommeil hypnotique «en fixant pendant quelques secondes un petit miroir métallique » (38). Cette pratique lui a permis de continuer à travailler et « d'économiser quinze années qui eussent [sinon] été perdues » (39) à dormir. En effet, dans la mesure où 
Monsieur Synthèse s'hypnotise «avec la volonté formelle de continuer, pendant [son] sommeil artificiel, à vivre intellectuellement comme à l'état de veille », il n'interrompt ni ses études ni le cours de sa vie quotidienne; et il ne ressent pas la moindre fatigue puisqu'il n'omet pas - simultanément - « suggère[r] à [son] corps l'idée de repos matériel » (39).

Tout ce qui pouvait paraître mystérieux, voire dangereux, à un observateur extérieur (le fait que le vieux savant ne s'alimente pas, ne dorme pas et ait fait l'acquisition de cinq cents scaphandres autonomes) est justifié par Monsieur Synthèse lors de son entretien initial avec le préfet de police. Chaque détail est expliqué, réinterprété et finalement ancré dans la sphère de la rationalité et de la complète scientificité. Comme le proclame Monsieur Synthèse haut et fort, il n'est « ni un sorcier, ni un mystificateur, mais un homme de science qui peut prouver tout ce qu'il avance » (36). Son esprit apparaît donc comme tout à fait sain, son seul défaut étant peut-être l'extrême clairvoyance qui fonde son indéniable supériorité.

Néanmoins, les preuves que Monsieur Synthèse apporte ne sont pas toutes du même ordre. Le processus de légitimation à l'œuvre dans la fiction repose

[p. 108]

sur des attendus plus complexes qu'il n'y paraît au premier abord et qui ne vont pas sans influer sur le diagnostic que l'on peut établir du fonctionnement et de l'étendue des pouvoirs attribués à l'esprit du principal protagoniste. Dans son roman, Boussenard se saisit en premier lieu de connaissances scientifiques avérées dont il fait la matière de son récit, œuvrant ainsi à vulgariser certaines données d'un savoir établi, par exemple, en ce qui concerne la composition chimique des aliments 5 . Une affiche-réclame diffusée début 1888 n'annonçait-elle pas la parution prochaine dans La Science Illustrée d'un "grand roman scientifique ${ }^{6} »$ ? Mais l'écrivain ne s'en tient pas là : il extrapole à partir de données scientifiques parcellaires et de résultats encore incomplets; il tire les conséquences d'avancées scientifiques décrites comme effectives dans la fiction alors qu'elles n'existent pas dans la réalité.

Premier exemple de ce mécanisme : à partir de connaissances confirmées portant sur la composition chimique des aliments, le romancier infère que le vieil homme lui-même, puis son équipage tout entier, peuvent se nourrir uniquement de pilules et renoncer à tout apport de nourriture traditionnelle. À l'instar de ses hommes, le maître d'équipage Pornic, bien que réduit au régime plus que spartiate de Monsieur Synthèse, se trouve "rassasié comme [s'il avait] double ration» (366).

Autre exemple: des premières expériences encourageantes portant sur la synthèse du carbone réalisées en 1880, qui n'ont cependant permis d'obtenir que des diamants de taille infinitésimale 7 , Boussenard infère que Synthèse peut 
posséder la faculté de produire «à volonté » des pierres précieuses artificielles "de toute grosseur» (25) qui sont présentées comme l'un des fondements de son immense fortune.

Troisième exemple : des expériences d'hypnose et de suggestion menées à Nancy et à la Salpêtrière sur des sujets le plus souvent porteurs d'une pathologie mentale, le romancier infère que Synthèse a la possibilité de rester 45 ans sans dormir naturellement et peut faire sombrer tout son équipage dans un semblable sommeil hypnotique des semaines durant.

Quatrième et dernier exemple : des principes de succession des espèces mis en évidence par la théorie évolutionniste darwinienne, Boussenard infère que Synthèse peut envisager de reproduire, en accéléré et à petite échelle, le processus qui a mené à l'apparition de l'homme sur la Terre.

Cette logique fictionnelle s'appuie sur un développement présumé à partir de l'état contemporain des connaissances scientifiques ; elle est caractéristique du roman d'anticipation, genre que Boussenard illustre avec Les secrets de Monsieur Synthèse. Or toutes ces extrapolations, dans la mesure où elles reposent sur un schéma identique, se confortent les unes les autres dans la fiction,

[p. 109]

des moins invraisemblables et déjà présentées comme réalisées dans la fiction (comme la production de pierres précieuses de synthèse) aux plus abracadabrantes, ces dernières n'ayant finalement pas moins de raisons que les précédentes de se réaliser dans la fiction, y compris quand elles consistent à réitérer en quelques mois le processus évolutif menant de la monère jusqu'à l'homme. La série de ces extrapolations homologues concourt enfin à confirmer, au sein de la logique fictionnelle ainsi définie, la santé mentale du personnage central.

Pourtant, en dépit de tous les éléments précédemment relevés, le doute ne tarde pas à s'installer dans l'esprit du lecteur : Monsieur Synthèse est-il si sensé que cela? Pour une grande part, l'intérêt du roman réside dans le jeu que Boussenard introduit entre les assertions et les indices de bonne foi scientifique du vieux savant et les failles qui se font progressivement jour dans son discours et son comportement.

Dès l'ouverture du roman, Monsieur Synthèse est soupçonné par le préfet de police qui vient l'interroger de n'être qu'un «monomane », prompt à « divaguer en enfourchant un dada qui a probablement emporté sa cervelle à travers les espaces planétaires » (27). Le vieil homme lui apparaît sous le masque de Darwin, on l'a vu, mais il est tout aussi important de noter que cette référence scientifique orthodoxe et valorisante est encadrée par deux autres qui le sont nettement moins. La nationalité suédoise du vieil homme le rapproche de " quelque mystique adepte de Swedenborg, [de] quelque rêveur à la cervelle obscurcie par les brumes 
natales » (19), tandis que sa bouche, en dépit de son grand âge «meublée de dents régulières parfaitement saines » (21), le fait ressembler à Victor Hugo, écrivain dont la proximité avec l'univers occulte n'est un mystère pour personne.

Surtout, le rapport que Monsieur Synthèse entretient avec la science paraît évoluer dans un sens peu compatible avec ses protestations initiales de rigueur scientifique. Au début, il assure n'être qu'un expérimentateur qui, disposant les éléments de la nature selon certaines procédures déterminées, peut amener des transformations. Il l'affirme explicitement :

- Je ne crée absolument rien.

Nul, ici-bas, ne peut faire de rien quelque chose, et pour l'homme le néant est toujours le néant!

Mais je combine les corps simples qui se trouvent partout dans la nature, mais j'utilise judicieusement les forces de la matière en partant du simple au composé, du général au particulier, de l'abstrait au concret. (35)

Voilà une profession de foi scientifique inattaquable que tout chercheur digne de ce nom ratifierait. Mais Monsieur Synthèse ne s'en tient pas là et ce

[p. 110]

paradigme de l'expérimentateur va se trouver progressivement parasité puis remplacé par celui du créateur, issu d'un tout autre univers. Souterrainement, deux modèles antithétiques se superposent et se combattent: celui de l'évolution et celui de la genèse.

Ainsi, avant même l'appareillage de tous les protagonistes pour la mer de Corail, Monsieur Synthèse explique à ses deux préparateurs, un chimiste et un zoologiste, qu'ils vont l'«aider puissamment à la création de ce sol vierge qui, à [son] ordre, va émerger de toutes pièces, du sein des eaux » (84). Si le souci de mettre en place un environnement d'expérimentation exempt de toute contamination et propice à la culture de la monère, ce " germe primordial » (442) qui va être récupéré par 5000 mètres de fond, caractérise une méthodologie scientifique maîtrisée et visant à « reproduire dans des proportions infiniment réduites, mais cependant identiques, les phénomènes de transformations lentement opérées pendant l'interminable succession des siècles » (130), il n'en va pas de même pour l'image parasite d'une terre qui apparaîtrait miraculeusement, ex nihilo, simplement en réponse à une parole de nature divine ou démiurgique, comme dans le texte biblique de la Genèse ${ }^{8}$. Mais on peut encore penser à ce moment de l'intrigue qu'il s'agit là d'une facilité de langage à laquelle cèderait le vieux chimiste, d'autant que lors de son entretien avec le pundit Krishna (on y reviendra), Monsieur Synthèse réitère son affirmation première : « Je ne veux rien créer... mais simplement transformer » (98). 
Néanmoins, une première faille explicite se fait jour dès ce moment, car le vieux savant continue en ces termes : «Créer !... Je ne le puis pas encore... du moins pour le présent » (98). S'il n'a pas encore cette faculté, il espère bien la posséder un jour. Et la retenue ne sera plus du tout de mise dès que Synthèse sera parvenu à bâtir les fondations de son île-laboratoire en stimulant artificiellement la croissance des madrépores. Prenant possession de l'îlot, «il s'écrie d'une voix retentissante » :

- Par ma volonté seule, les forces de la nature se sont mises en travail et la terre est sortie du sein des eaux!

À ma voix cette terre neuve, stérile encore, se peuplera d'organismes vivants !

Toute la série des êtres, évoluera ici, depuis la monère, la cellule primitive, jusqu'à l'homme lui-même.

Ici la vie apparaîtra comme autrefois sur la terre! Les espèces naîtront, se transformeront et périront, pour renaître, se transformer, périr encore, et s'absorber dans l'homme primordial, l'homme type, sans ascendants, l'homme de la Synthèse !

Tel un explorateur prenant possession d'un nouveau continent, Synthèse déploie alors un étendard sur lequel des

[p. 111]

diamants, enchâssés dans la substance même du pavillon, sont juxtaposés de façon à composer des lettres.

Ces lettres forment trois mots...

Une devise que les deux Français lisent avec stupeur, car sa signification leur permet enfin de mesurer l'envergure de leur maître.

ET EGO CREATOR. (127-28)

Cette devise (qui apparaît sous la forme d'un calligramme dans le texte même de l'ouvrage) sera ironiquement rappelée à la toute fin du roman, lorsque Monsieur Synthèse échouera - de peu, pense-t-il - dans la réalisation de son projet :

- Un enfant... un noir !

Un peu plus tard... l'enfant était un homme... un nègre.

Plus tard... encore... l'homme était un blanc...

L'évolution... le Grand-CEuvre... transformation...

Je pouvais dire : Et ego Creator ! (480)

Ce modèle génésiaque avorté est volontairement combiné par Boussenard à celui de l'alchimie, ce qui contribue encore un peu plus à brouiller les pistes. Déjà, le titre du roman et l'utilisation du terme «secrets » aiguillaient la lecture vers un tout autre domaine que celui de la science : un savant n'a pas de secrets; il formule des hypothèses qu'il s'ingénie à vérifier afin que, s'il y parvient, elles se muent en 
vérités. Il cherche à percer les secrets et non à les conserver. Au contraire, Monsieur Synthèse refuse d'expliquer au préfet de police en quoi consiste son projet : «Quant à l'usage auquel je destine cette terre [...], c'est mon secret » (42), lui assène-t-il. De même, on ne connaîtra jamais le « secret» qui aurait permis au vieux savant, dans son entreprise, de « remplacer cette influence des millions de siècles qui ont modifié [...] les êtres primitifs » (240).

Cette prégnance du secret préfigurant l'altération progressive des procédures scientifiques initialement promues se retrouve dans le nom que Monsieur Synthèse a choisi de donner à son ultime projet : le « Grand-CEuvre ». En alchimie, cette expression désigne la pierre philosophale, substance hypothétique ayant le pouvoir de transmuter les métaux et d'apporter l'immortalité à qui la trouvera. Or c'est bien à une entreprise proche que se livre - de fait - Monsieur Synthèse, alors même que le narrateur caractérise le savant comme ayant su préalablement « retrouver dans le fatras de l'alchimie des vérités pressenties par les anciens, mais incohérentes et reposant sur des hypothèses déraisonnables, et les appuyer sur des lois aujourd'hui bien connues» (442). En effet, si le vieux savant aspire à reproduire en laboratoire l'évolution des espèces, ce n'est pas pour prouver la pertinence théorique et pratique de la

[p. 112]

thèse de Darwin ; c'est pour obtenir, une fois et une seule, un résultat particulier, sous la forme d'un homme totalement vierge, qui permettra au vieillard, d'une certaine manière, d'atteindre à l'immortalité puisque cet homme deviendra l'époux - aimant et dénué de toute tare physique ou morale - de sa petite-fille. Le but poursuivi est de rendre impossible la réitération de la catastrophe qui a autrefois frappé la propre fille de Monsieur Synthèse, cyniquement délaissée par son mari et morte en couches :

Puisque la suggestion est toute puissante sur des êtres tels que les ont faits l'éducation, le milieu, l'hérédité, la maladie même, à plus forte raison ses manifestations devraient être irrésistibles, permanentes chez un être complètement neuf, vierge de toute impression.

Telle était donc la conception du Grand-CEuvre. (443)

D'autres passages du roman font explicitement écho à l'univers de l'alchimie tel qu'il a été revisité par l'imaginaire du XIX ${ }^{\mathrm{e}}$ siècle $^{9}$ et renforcent sa présence sous-jacente agissante. Par exemple, le préparateur chimiste Alexis Pharmaque, qui est le protagoniste qui soutient avec le plus de conviction l'entreprise de son patron, «semble sortir d'un de ces laboratoires bourrés de cornues, de matras, d'appareils baroques, de crocodiles empaillés où les alchimistes du Moyen-âge élaboraient leurs sorcelleries » (11). 
L'entreprise scientifique se trouve ainsi brouillée et dévoyée : toute idée de reproductibilité est abandonnée, l'expérience conçue par Monsieur Synthèse étant par définition unique; et la dimension très personnelle du projet se révélant finalement prioritaire, celui-ci apparaît inexorablement grevé par des affects parasites. Ainsi, lorsque le préparateur chimiste Alexis Pharmaque conçoit quelques doutes sur la bonne marche du Grand-CEuvre, il n'ose pas avertir Monsieur Synthèse : «Le Maître, avec sa manie de chercher partout la petite bête, serait capable d'en faire une maladie » (392).

Cause et/ou conséquence de tout cela, l'esprit de Monsieur Synthèse ne ressort effectivement pas indemne de ce processus de brouillage ; la raison du vieil homme apparaît de plus en plus chancelante au fur et à mesure que se déploie la fiction. Les deux auxiliaires du savant observent à plusieurs reprises des signes cliniques morbides inquiétants, en particulier un "commencement de gangrène sénile » (140), à l'origine d'un « ramollissement cérébral, produit par l'ossification des artères de la base du crâne » (205). Surtout, Monsieur Synthèse s'abîme de plus en plus profondément dans la contemplation de son œuvre :

Absorption! C'est bien là le mot applicable à Monsieur Synthèse qui prend automatiquement sa nourriture scientifique, s'hypnotise et s'éveille par habitude, oublie le monde entier, ne voit plus

[p. 113]

rien au-delà de son laboratoire, ce microcosme qui est, à notre monde, ce que la goutte d'eau est à l'Océan. (285)

Le caractère pathologique de cette attitude ne fait plus de doute à la fin du roman lorsqu'elle atteint le stade d'une complète hébétude, d'une " désespérante atonie » (484). D'ailleurs, bien avant que la raison de Monsieur Synthèse ne finisse par sombrer, l'aide zoologiste Roger-Adams avait posé un diagnostic de folie englobant son patron et son collègue Alexis Pharmaque : "Ils enfourchent le même dada, poursuivent la même chimère, proposent les mêmes hérésies et courent audevant des mêmes déboires. Nul doute que, avant seulement trois mois, ils ne soient fous à lier » (242).

La figure de Synthèse rejoint ici celle du savant fou, réactivant le mythe de Prométhée, et sa version moderne, le mythe de Faust ${ }^{10}$. Transgressant les prérogatives de Dieu ou de la nature, le savant veut créer de toutes pièces un être vivant :

Cet homme, pour lequel il le croyait du moins, la nature épuiserait tous ses trésors, cet homme dont il aurait provoqué, puis surveillé heure par heure la genèse, qui serait sa créature à lui, sa chose, son œuvre, deviendrait l'époux de son enfant. 
[...] cet homme serait ce qu'il voudrait, puisqu'il recevrait de lui la pensée, quand, nouveau Prométhée, il l'animerait d'un souffle, et lui suggérerait, dès la première minute de sa vie, l'idée dominante d'affection, de dévouement, d'amour, en un mot, pour la jeune fille. (443)

Il est cependant excessivement rare dans le cours du roman que le lecteur ait ainsi accès aux pensées de Monsieur Synthèse par l'intermédiaire du discours du narrateur omniscient. La plupart du temps, il n'a qu'une connaissance très partielle des pensées et de l'état de conscience du principal protagoniste : le plus souvent, le narrateur décrit seulement des attitudes («Monsieur Synthèse manifest[e] quelques signes extérieurs de préoccupation » [454]) ; d'autres fois, il rapporte des paroles mais se déclare dans l'incapacité d'expliquer certaines actions ou certains faits dont il délègue l'interprétation, plus ou moins éclairante selon les moments, à des personnages de la fiction :

A-t-il enfin l'intuition de l'avenir ?... prévoit-il que ce voyage est le salut de son enfant? [...]

C'est ce que ses familiers, étonnés tout d'abord, et qui, somme toute ont bien autre chose à faire, ne se donnent pas la peine d'approfondir. (285)

L'auteur a aussi recours aux possibilités offertes par la présence d'un policier qui surveille le savant durant toute l'intrigue: les deux rapports que l'agent envoie à son supérieur hiérarchique fournissent au lecteur des

[p. 114]

informations que le narrateur ne lui a jamais communiquées et qui expliquent certains faits incompréhensibles autrement. Grâce à cette gestion rusée des points de vue, Boussenard parvient à conserver entier le mystère sur l'état de conscience et la santé mentale réels de Monsieur Synthèse tout au long du roman. Le lecteur assiste, impuissant, au dépérissement d'un esprit qui faisait d'abord montre d'une complète maîtrise sur son environnement, avant de perdre progressivement pied jusqu'à complètement sombrer: "Hissé par les deux sauveteurs, le Maître, semblable à un titan foudroyé, insensible à tout, contemple d'un air égaré ce sol sorti à son ordre du sein des flots » (479). Le procédé est d'autant plus intéressant à souligner que Boussenard en prend l'exact contrepied dans Dix mille ans dans un bloc de glace. Dans ce roman présenté comme l'épilogue des Secrets de Monsieur Synthèse, on ne quitte quasiment pas le point de vue du - dorénavant - assurément très vieux savant.

L'ultime entreprise de Monsieur Synthèse, son Grand-CEuvre, se solde (vraisemblablement) par un échec. L'esprit du savant n'a pas su conserver intacts les principes scientifiques qui l'animaient autrefois; il les a laissés vicier par ses affects, en particulier par l'amour paternel qu'il porte à sa petite-fille orpheline. La 
conception même d'un projet qui ambitionnait, en reproduisant la succession des espèces dans une île-laboratoire, de créer un homme destiné à servir d'époux et qui serait vierge de toute empreinte montre que l'esprit de Monsieur Synthèse était affecté depuis longtemps. Mais Boussenard a eu l'intelligence scénarique de retenir longtemps ces informations, jusqu'à ce que l'esprit de son personnage apparaisse déjà au lecteur comme irrémédiablement atteint.

Dans la fiction, l'extension du domaine du merveilleux n'aura donc pas lieu. $\mathrm{Au}$ contraire, le roman illustre la forte et générale aspiration de la science du XIX ${ }^{e}$ siècle à la scientification du merveilleux. Dans la mesure où Monsieur Synthèse pratique l'hypnose et la suggestion avec un succès que rien ne dément jamais, la fiction les intègre dans un ensemble de pratiques validées et efficaces, entérinées par les expérimentations reconnues d'Hippolyte Bernheim et de Jean Martin Charcot, qui ont fait l'objet de publications scientifiques dont les références sont à plusieurs reprises indiquées avec précision par l'auteur en note de bas de page.

Néanmoins, si le roman ne promeut pas un merveilleux qui résulterait d'une science dévoyée, il s'attache à interroger la résistance dont fait preuve le merveilleux lorsque la science échoue encore à expliquer la survenue de faits mystérieux. Dans le roman, ce questionnement est porté par l'énigmatique personnage nommé Krishna qui apparaît inopinément dans la cabine du navire de Monsieur Synthèse, en plein milieu de la mer de Corail, pour le

\section{[p. 115]}

mettre en garde contre la réalisation du Grand-CEuvre. Vêtu à la façon des Hindous, cet être qui s'apparente à un esprit dans sa manière d'apparaître et de se déplacer, est un "pundit, un adepte » (99). Le vieux savant n'est pas inquiété par l'arrivée de celui qu'il considère comme un ami et dont il persiste à expliquer les étonnants pouvoirs par une simple sensibilité hors du commun, une "pénétration extraordinaire » (96). Dans leur dialogue, deux systèmes s'opposent : il y a d'une part les Occidentaux qui, selon Krishna, ont tort de "rechercher la science au moyen de [leurs] sens corporels, parce qu'ils sont loin d'être infaillibles, et que leur action est forcément limitée »; et d'autre part les Orientaux qui, selon Monsieur Synthèse, "prétend[ent] [s']assimiler toute la science, toute la puissance qu'un être humain est susceptible de posséder, par les jeûnes prolongés, les méditations, la tension rigoureuse des facultés intellectuelles vers le but à atteindre » (99).

Si Monsieur Synthèse accepte d'envisager qu'un jour les deux systèmes puissent être conjoints, le pundit essaye quant à lui de convaincre son interlocuteur que la méthode qu'il suit le conduit à sa perte et qu'il est urgent pour lui d'en changer : " un péril [...] menace ton existence ou ta raison » (95). Krishna redoute les conséquences de l'expérimentation prévue par Synthèse non en ce 
qu'elle pourrait déboucher sur un succès, mais en ce qu'elle perturbe le cours naturel des choses et entraînera un jour la perte du savant : "Je te l'ai dit tout à l'heure, ta raison et ta vie sont menacées » (101). Après la disparition du pundit, Monsieur Synthèse tente d'expliquer cette curieuse entrevue par une hallucination due au sommeil hypnotique dans lequel il était alors plongé. Néanmoins,

il ne peut retenir un geste d'étonnement, à l'aspect d'un objet laissé comme à dessein sur le tapis.

Cet objet, qu'il reconnait parfaitement, est une des deux babouches qui chaussaient les pieds du pundit. (102)

La tentative de Monsieur Synthèse pour ramener l'apparition de KrishnaCendrillon dans les limites de la science telle qu'elle est redéfinie au sein même de la fiction achoppe sur cette pantoufle. L'hypnose ne peut expliquer sa présence. L'esprit du savant, pourtant alors au faite de sa clarté, échoue à rendre compte de ce phénomène et c'est peut-être là, déjà, le tournant de l'histoire. L'obscurcissement progressif de la raison du savant se confirmera ensuite au fur et à mesure que les étapes du Grand-OEuvre s'enchaîneront sans qu'on sache jamais vraiment quelle en est la cause exacte. Il faut d'ailleurs sûrement plutôt parler d'un faisceau de causes qui pourraient être la perversion du processus scientifique par des affects individuels, la réalisation de la prédiction de Krishna, ou encore l'abus de l'hypnose. Dans son ouvrage

\section{[p. 116]}

intitulé Le spiritisme, cité par Boussenard dans son roman, le docteur Paul Gibier «signal[e] le péril inhérent aux expériences de psychisme avec lesquelles on joue cependant sans se douter du grand danger qu'elles font courir ${ }^{11}$ ». L'utilisation excessive du procédé pourrait donc présenter des effets secondaires délétères se traduisant par une altération progressive de la conscience.

Si le roman Les secrets de Monsieur Synthèse est certes représentatif de cette tendance lourde de la littérature de vulgarisation scientifique de la fin du $\mathrm{XIX}^{\mathrm{e}}$ siècle qui vise à réduire l'empan du merveilleux, on a tenté de montrer ici, en suivant les méandres de l'esprit de son personnage principal, qu'il le faisait d'une manière rusée et décalée, dans un récit foisonnant et complexe qui, tout en intégrant certaines dimensions du merveilleux à la science, s'ingénie pourtant à lui ménager une place persistante - peut-être pour qu'il lui serve d'aiguillon ?

Néanmoins, l'angle d'attaque choisi n'a permis de développer que deux des dimensions en jeu dans ce roman, en passant sous silence les forces de la nature qui se déchaînent à plusieurs reprises et, surtout, les menées subversives des ennemis de Monsieur Synthèse, c'est-à-dire les vengeances imbriquées du prince hindou dépossédé par Synthèse et du frère de son gendre indigne, sur lesquelles se 
greffe la mystification fomentée par le cupide préparateur Roger-Adams. C'est seulement en les prenant en compte dans leur pluralité et dans leurs complexes intrications qu'on pourra avoir un panorama véritablement complet de la reconfiguration inattendue qu'opère ce roman atypique ${ }^{12}$.

CNRS, UMR 5317 IHRIM

(Institut d'histoire des représentations et des idées dans les modernités)

\title{
Notes
}

\begin{abstract}
${ }^{1}$ Louis-Henri Boussenard (1847-1910) est un écrivain français surtout connu pour ses romans d'aventure et, en particulier, pour son personnage reparaissant du « gamin de Paris ». La plupart de ses nombreux romans ont été prépubliés en feuilleton dans la presse (dans le Journal des Voyages, Le Figaro ou Le Petit Parisien) et lui valurent une célébrité aujourd'hui passée. Le roman a paru d'abord en 49 livraisons dans La Science Illustrée entre le 10 mars 1888 et le 9 février 1889, puis, en volume, dès la fin de l'année 1888. L'ouvrage (Paris: C. Marpon et F. Flammarion, [s.d.]) est disponible sur Gallica (http://gallica.bnf.fr/ark:/12148/bpt6k123103x). Il comprend aussi Dix mille ans dans un bloc de glace. Les références paginales renvoient à cette édition.
\end{abstract}

${ }^{2}$ Voir Joseph-Pierre Durand, Le merveilleux scientifique (Paris : Félix Alcan, 1894). L'ouvrage est disponible sur Gallica (http://gallica.bnf.fr/ark:/12148/bpt6k6342148t).

${ }^{3}$ «Monsieur Synthèse, dernier descendant d'une longue et illustre généalogie de savants, héritier de leurs traditions, de leurs travaux, de leurs découvertes, pensait de très bonne foi que rien n'est impossible à la science » (442).

[p. 117]

${ }^{4}$ Voir Dix mille ans dans un bloc de glace, court roman publié par Louis Boussenard après Les secrets de Monsieur Synthèse et qui en propose une suite.

${ }^{5}$ Dans La Science Illustrée, où le roman paraît initialement, la composition chimique des aliments fait d'ailleurs l'objet d'une série d'articles concomitante sous le titre : "La science familière et usuelle ». Voir Florence Carneiro et Brigitte Rozet, «Quelques aspects de la science dans le roman », in La science pour tous : Sur la vulgarisation scientifique en France de 1850 à 1914, Bruno Béguet, éd. (Paris : Bibliothèque du Conservatoire national des arts et métiers, 1990), 125 sq.

6 Voir l'estampe-lithographie conservée à la BnF, disponible sur Gallica (http://gallica.bnf.fr/ark:/12148/btv1b9013810n), dont voici le texte : "La Science Illustrée / très beau journal illustré / commence la publication de / Les secrets de Monsieur Synthèse / grand roman scientifique / par Louis Boussenard / auteur du Tour du monde d'un gamin de Paris ».

${ }^{7}$ En 1880, le chimiste écossais James Ballantyne Hannay (1855-1931) a annoncé avoir synthétisé le diamant.

${ }^{8}$ Notons d'ailleurs la mention d'une arche de Noé dans le cours du récit: "Là vivaient généralement des reptiles, des sauriens, des crustacés dont la rencontre faisait pâmer d'aise notre savant qui collectionnait à outrance les sujets vivants et les embarquait dans notre chaloupe transformée bientôt en une véritable arche de Noé » (429). 
${ }^{9}$ Rappelons que si l'alchimie avait été frappée de péremption par la révolution opérée par Antoine de Lavoisier au XVIIIe siècle, elle n'en restait pas moins alors une science sérieuse quoique révolue ; $c^{\prime}$ est le $\mathrm{XIX}^{\mathrm{e}}$ siècle qui a introduit une relecture ésotérique et occultiste de l'alchimie et a permis l'émergence de l'alchimiste comme figure du savant fou, dont Monsieur Synthèse est porteur de plusieurs traits. Voir Bernard Joly, «La figure de l'alchimiste dans la littérature du XIXe et du $\mathrm{XX}^{\mathrm{e}}$ siècle. Savant fou ou folies scientifiques : De l'alchimie à la chimie », in Le savant fou, Hélène Machinal, éd. (Rennes : Presses universitaires de Rennes, 2013), 75-88.

10 Et Frankenstein n'est pas loin.

11 Paul Gibier, Le spiritisme (fakirisme occidental) : Étude historique, critique et expérimentale (Paris : O. Doin, 1896), 386.

12 Voir Stéphanie Dord-Crouslé, «Une simple expérience de laboratoire? Fiction hybride et mélange des genres dans Les secrets de Monsieur Synthèse par Louis Boussenard », communication prononcée au 41 $\mathrm{e}$ Congrès de Nineteenth-Century French Studies (2015), à paraître dans la revue Arts et Savoirs, http://aes.revues.org/. 